\title{
Generation of small-compound libraries via random chemistry by Fenton's reagent
}

\author{
Eva Kugelmann and Ulrike Holzgrabe* \\ Institute of Pharmacy and Food Chemistry, University of Würzburg, Am Hubland, \\ 97074 Würzburg, Germany \\ E-mail: u.holzgrabe@pzlc.uni-wuerzburg.de
}

\begin{abstract}
Re-combinatorial chemistry, an auspicious method for generating small-compound libraries by means of $\gamma$-irradiation of active lead compounds, disclosed the formation of new compounds with reassembled or rearranged structures mainly to be derived from radical chemistry. Therefore, Fenton's reagent was applied to a methanol/water solution of tacrine to mimic the irradiation. In fact, these preliminary studies revealed the generation of a small compound library with a product composition similar to those obtained by $\gamma$-irradiation.
\end{abstract}

Keywords: Re-combinatorial chemistry, random chemistry, Fenton's reagent

\section{Introduction}

We have recently reported a new method for the serendipitous generation of a small-compound library of biologically active substances by means of $\gamma$-irradiation of active compounds or pharmacophoric fragments of active compounds or mixtures of fragments. The concept of the socalled "re-combinatorial chemistry" (random chemistry) ${ }^{1}$ proved valuable for the development of new inhibitors of the acetylcholinesterase ${ }^{2}$ as well as antiplasmoidal and antiviral compounds. ${ }^{3}$ However, most of the new compounds formed by $\gamma$-irradiation are products formed by radical chemistry. Especially solvent free radicals formed by $\gamma$-irradiation seem to be the main players in the formation of new compounds. Thus, reagents initiating the formation of radicals should be applicable in "re-combinatorial chemistry".

Since the $\gamma$-irradiation is a highly sophisticated and expensive method in this study we were aiming for the proof-of-principle. We checked whether Fenton's reagent, one of the best known, cheapest and easiest to handle one electron transfer reagents, can serve as an initiator of the production of a small compound library of active substances. The inhibitor of the acetylcholinesterase, tacrine (1), was chosen as a model compound because the irradiation of tacrine has been already extensively studied. ${ }^{2}$ Hence, the findings of the irradiation and the 
oxidation with Fenton's reagent can be easily compared. Since Fenton's reagent is not very soluble in organic solvents, mixtures of water and organic solvents had to be applied.

\section{Results and Discussion}

The irradiation of tacrine with $\gamma$-rays derived from a ${ }^{60} \mathrm{Co}$ source at room temperature led to the formation of a considerable library with a multitude of different compounds. The experiments were performed with solutions of tacrine hydrochloride in water and various alcohols, respectively. Comparison of the product spectra from the different solvents by means of RPHPLC revealed methanol and water solutions to contain the highest number and concentration of new products (Fig. 1). After bioassay-guided HPLC fractionation compounds with very high inhibitory activity against AChE could be separated in both cases and their structures elucidated.

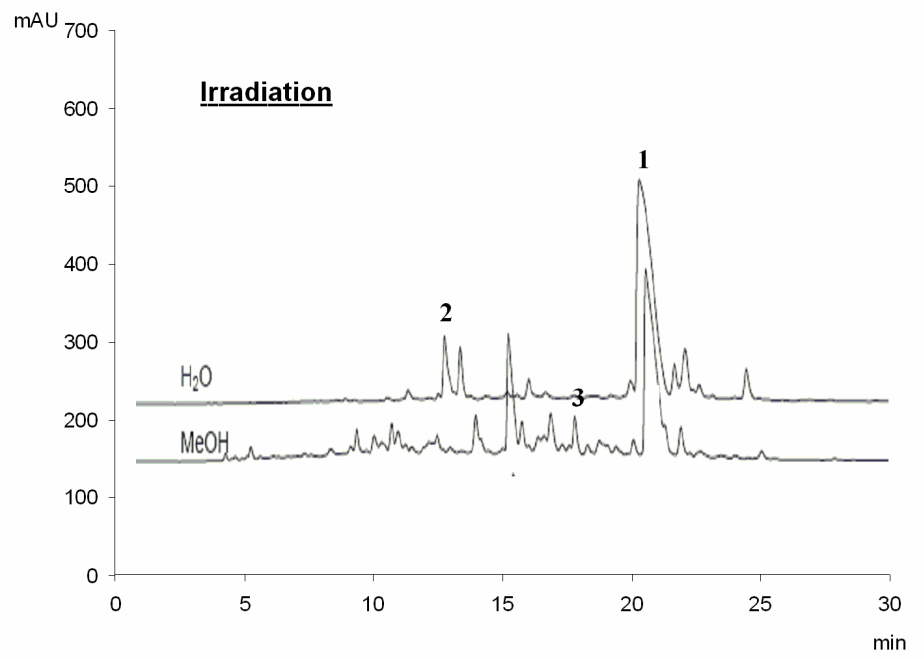

Figure 1. HPLC separation of irradiated tacrine in water or methanol; 1-hydroxytacrine (2), $\operatorname{ATAM}(\mathbf{3})$, tacrine (1). (For chromatographic protocol see Experimental section). ${ }^{2}$

Upon irradiation of tacrine in water, 1-hydroxytacrine (2), a known active metabolite of tacrine, was found. On irradiation of tacrine in methanol however, a new hydroxymethylated tacrine of high activity, named ATAM = (9-amino-5,6,7,8-tetrahydro-acridin-4-yl)-methanol (3), was identified. ${ }^{2}$ 
<smiles>Nc1c2c(nc3ccccc13)CCCC2</smiles>

1<smiles>Nc1c2c(nc3ccccc13)CCCC2O</smiles>

2<smiles>Nc1c2c(nc3c(CO)cccc13)CCCC2</smiles>

3

These compounds affirm the assumption that the radiolysis of water mainly results in hydrogen atoms $(\mathrm{H})$, hydroxyl radicals $(\cdot \mathrm{OH})$ and hydrated electrons $\left(\mathrm{e}^{-}\right.$aq $)$whereas in methanol accessory secondary radiolysis products are formed, e.g. a $\cdot \mathrm{CH}_{2} \mathrm{OH}$ radical, which is probably responsible for the formation of ATAM. ${ }^{4}$

As solvent free radicals are obviously the main players in the formation of new compounds the idea arises that these radicals can be formed either by $\gamma$-irradiation or by radical forming agents such as Fenton's reagent. To verify this assumption we ascertained if Fenton's reagent is applicable to "re-combinatorial chemistry".

Thus, an aqueous $\mathrm{H}_{2} \mathrm{O}_{2}$ solution was added to a solution of ferrous sulfate and tacrine in a methanol/water mixture. The methanol/water mixture was chosen for two reasons, on the one hand due to the high solubility of tacrine hydrochloride in water, methanol and ethanol and the poor solubility of Fenton's reagent in organic solvents and on the other hand because the results should be comparable to those obtained by $\gamma$-irradiation in water and methanol systems.

The preliminary studies revealed the generation of a small compound library and in fact a product composition similar to those obtained by $\gamma$-irradiation in methanol and water (cf Fig. 1 and Fig. 2). Both 1-hydroxytacrine and ATAM could be identified.

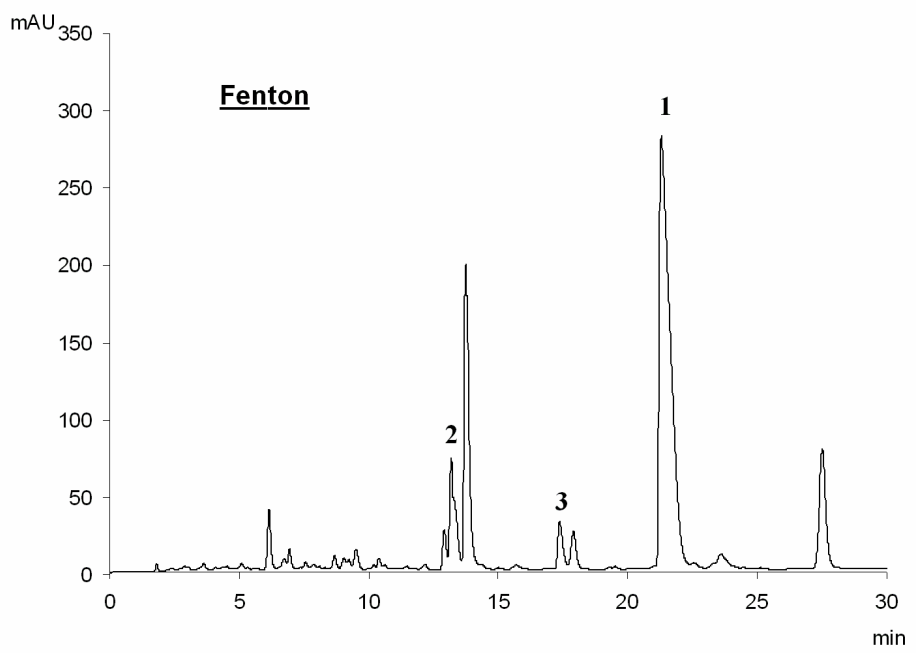

Figure 2. HPLC separation of with Fenton's reagent converted tacrine in a water/ methanol mixture; 1-hydroxytacrine (2), ATAM (3), tacrine (1). (For chromatographic protocol see Experimental section). 
To separate the obtained library first an analytical RP-HPLC method with a gradient elution was used. Therefore the composition of the mobile phase was changed continuously by increasing the content of organic solvent from $10 \%$ to $40 \%$ in 30 minutes. With this procedure good separations of the compound libraries could be achieved in an acceptable period of time. By means of LC/MS analysis the main peaks could be assigned to the compounds 1hydroxytacrine (2), ATAM (3) and tacrine (1). Additionally, the peak purity of each peak could be confirmed.

The great advantage of the application of Fenton's reagent is the fact that it is possible to vary the solvent as well as other reaction conditions in a wide range in order to influence product composition. When exploring limits and possibilities, differences in product composition were detected.

First the concentration of hydrogen peroxide was varied and found to have great impact. At hydrogen peroxide concentrations less than 100-fold excess in comparison to tacrine no reactions could be observed. A 200-fold excess of hydrogen peroxide however led to a notable conversion and distinct yields of ATAM (3) and 1-hydroxytacrine (2), whereas increasing the excess to 300 had no further effect. Thus, a 200-fold excess of Fenton's reagent was found to be necessary to provide a satisfying conversion of tacrine.

The raise of reaction time from 2 to $24 \mathrm{~h}$ gained no advantages. Thus, it can be concluded that under these conditions, reaction time is shorter than $2 \mathrm{~h}$.

Trials to increase the methanol concentration to more than $15 \%$ failed, as ferric sulfate precipitates and the yields decreased.

In order to exclude a possible influence of atmospheric oxygen on the reaction, one experiment was carried out under nitrogen atmosphere. However, no changes of the composition of the developed compound library appeared.

The addition of EDTA had no effect on any yields although an increase of yields is often described in literature in similar cases. Only the combination of EDTA addition and increasing the reaction time led to a slight increase of the yields of 1-hydroxytacrine (2).

The main influence on product composition however showed the variation of $\mathrm{pH}$ (cf Fig. 3). A decreasing $\mathrm{pH}$ value results in an increasing conversion of tacrine. Decreasing the $\mathrm{pH}$-value from 5 to 4 and 2 increased the yields of the ATAM (3) and an unknown product (4), whereas at pH 5 the highest yields of the 1-hydroxytacrine (2) were obtained. 

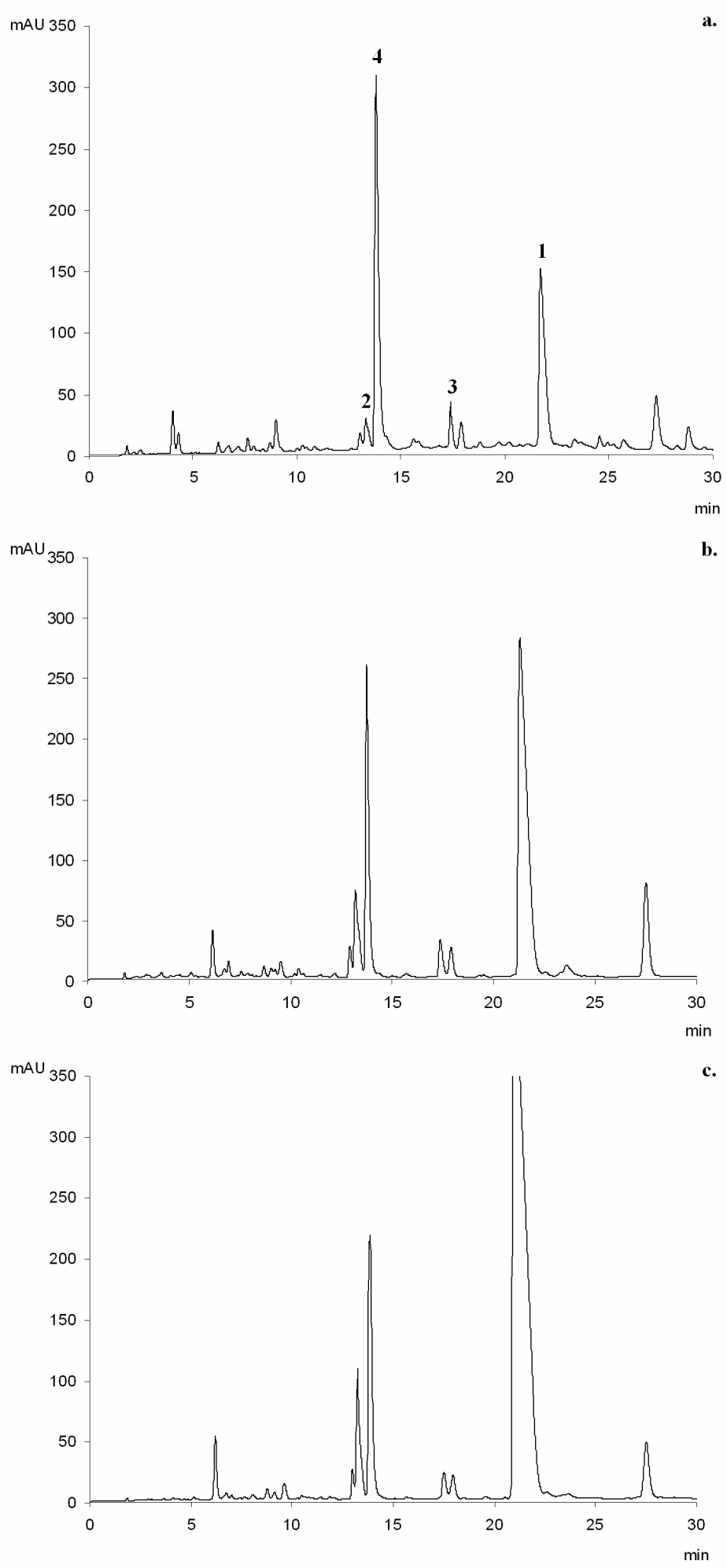

Figure 3. HPLC chromatogram of the treatment of tacrine with Fenton's reagent in a mixture of methanol and water at $\mathrm{pH} 2$ (a), $\mathrm{pH} 4$ (b) and $\mathrm{pH} 5$ (c). (For chromatographic protocol see Experimental section). 
To determine the structure of the unknown product (4), the experiment was performed at $\mathrm{pH}$ 2 followed by a semi-preparative HPLC separation combined with a solid-phase extraction (C18) with methanol as mobile phase for up-concentration and for isolation of the compound. For the semi-preparative HPLC separation a slightly modified version of the analytical HPLC method was used. The same solvents were applied, but a stepwise not a continuous change of the composition of the organic solvent had to be employed for isolation purpose. Accompanying $\mathrm{LC} / \mathrm{MS} / \mathrm{MS}$ experiments were performed in order to collect structural information.

The tandem mass spectrometry experiments showed that compound $\mathbf{4}$ possesses a mass-tocharge ratio of $\mathrm{m} / \mathrm{z}=213$ and reacted to a product ion with a $\mathrm{m} / \mathrm{z}$ ratio of $\mathrm{m} / \mathrm{z}=197$. In literature the loss of 16 amu corresponds either with the loss of an amino group $\mathrm{NH}_{2}{ }^{+}$or with the loss of an oxygen atom $\mathrm{O}^{+}$. Beyond it $\mathrm{MS}^{3}$-measurements exhibit the formation of an $\mathrm{m} / \mathrm{z}=182$ product ion (Fig. 4). This fragment is equivalent to the unsubstituted 1,2,3,4-tetrahydroacridine skeleton which is also observed in MS/MS experiments of tacrine (1) and 1-hydroxytacrine (2). ${ }^{2}$

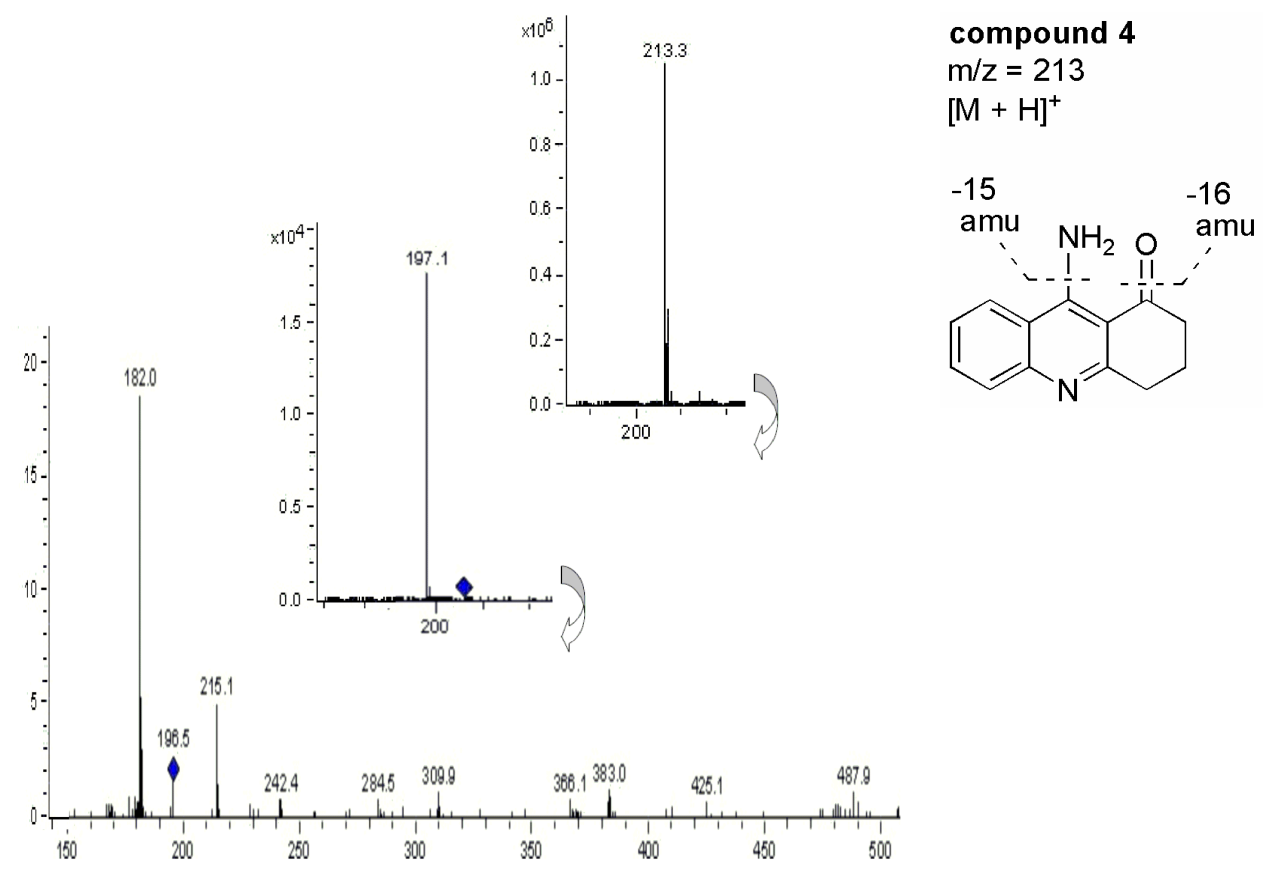

Figure 4. $\mathrm{MS}^{2}$ - und $\mathrm{MS}^{3}$-fragmentation of compound $\mathrm{m} / \mathrm{z}=213$.

Beside the fact, the MS measurements already indicate a tacrine skeleton with a carbonyl group, the NMR data affirmed the assumption. The ${ }^{13} \mathrm{C}-\mathrm{NMR}$ spectra and the four ${ }^{1} \mathrm{H}-\mathrm{NMR}$ signals for hydrogen $\mathrm{H}-5, \mathrm{H}-6, \mathrm{H}-7$ and $\mathrm{H}-8$ indicated an intact aromatic tacrine scaffold since they have nearly the same chemical shifts as in tacrine. However, only six aliphatic protons remained with two triplets at 3.24 and $2.67 \mathrm{ppm}$ and one triplet of triplets at $2.16 \mathrm{ppm}$ indicating a substitution on the cyclohexyl ring of tacrine in position 1 or 4 . A ${ }^{13} \mathrm{C}$ signal at $199.5 \mathrm{ppm}$ 
indicates furthermore the existence of a carbonyl keto group. The synthesis of an oxidized tacrine in position 1 and the comparison of the NMR and MS spectra finally confirmed the structure of 9-amino-3,4-dihydroacridin-1(2H)-one (4).

This tacrine derivative is already known as precursor in the syntheses of 1-hydroxytacrine. ${ }^{5}$ Nevertheless no testing of this compound of the biological activity, i.e. of its possibility to inhibit acetylcholinesterase has been published so far. Hence, compound $\mathbf{4}$ was subjected to a modified Ellman's test ${ }^{6}$ in order to check the inhibitory activity towards AChE. 4 inhibited AChE halfmaximally at $\mathrm{IC}_{50}=319 \mathrm{nM}$ which is about three times higher than for 1-hydroxytacrine $\left(\mathrm{IC}_{50}=\right.$ $106 \mathrm{nM}$ ) and about 7 times higher than for tacrine $\left(\mathrm{IC}_{50}=44 \mathrm{nM}\right)$, respectively.

Examination of X-ray crystal structure of tacrine ${ }^{7}$ revealed the 4-aminoquinoline portion of the tacrine molecule to be responsible for the binding of the drug to the enzyme. Tacrine is stacked near the bottom of the gorge against the rings of Trp84 and Phe330, the ring nitrogen is hydrogen bonded to the main-chain carbonyl oxygen of His440, and the amino nitrogen forms a hydrogen bond to a water molecule. ${ }^{8}$ The cyclohexyl ring however acts mostly as a block for the substrate at the active site. ${ }^{9}$ The introduction of a carbonyl group might lead to a steric hindrance worsening drug binding and thus be responsible for the readily increased $\mathrm{IC}_{50}$ values.

\section{Conclusions}

The easy, inexpensive and fast handling is the main advantage of the application of Fenton's reagent in "re-combinatorial chemistry". The limited solubility of Fenton's reagent in organic solvents however is a significant disadvantage as well as the poor solubility of organic compounds and potential drugs in aqueous solutions. However, since drugs in general should exhibit a high water solubility to ensure bioavailability the limitation can be an advantage at the same time as the products formed under these conditions are likely to be water soluble.

Taken together, the findings of the application of Fenton's reagent in "re-combinatorial chemistry" confuted the assumption of Ploszynska et al. ${ }^{10}$ that reactive species generated in Fenton's system are not the same as the species generated during water radiolysis, and revealed the method to be a pilot experiment for $\gamma$-irradiation and thus, to be a powerful tool for the generation of small-compound libraries in drug discovery.

Additionally, it may serve as a screening method for the metabolic profile of a new biologically active compound because the underlying chemistry of both reactions Fenton's one electron oxidation and cytochrome P450 oxidations, being the main metabolism enzyme, are similar. 


\section{Experimental Section}

General Procedures. Tacrine was synthesized according to refs 11. Solutions of tacrine were prepared in solvents of analytical grade and solvents used for LC, LC/MS and LC/MS/MS measurements were HPLC grade throughout. HPLC and analytical grade methanol were purchased from Merck (Darmstadt, Germany) and ammonium acetate, ferrous sulfate, formic acid, hydrogen peroxide, salts, and reagents for the Ellman test were from Fluka (Taufkirchen, Germany). HPLC grade water prepared by means of the Milli-Q purification system (Millipore, Eschborn, Germany) was used throughout.

$\boldsymbol{\gamma}$-Irradiation of tacrine. Solutions of tacrine hydrochloride $(2.34 \mathrm{mg} / \mathrm{mL})$ in water and methanol were exposed to $\gamma$-rays from a ${ }^{60} \mathrm{Co}$ source as described in ref. 2 .

Conversion with Fenton's reagent. A solution of $10^{-4} \mathrm{~mol}$ ferrous sulfate in sulfuric acid and $10^{-4}$ mol tacrine in a methanol/water (15:85) mixture was converted with a aqueous $\mathrm{H}_{2} \mathrm{O}_{2}$ solution (35\%) and stirred for $2 \mathrm{~h}$ at room temperature. After evaporation the solvent the residue was purified by column chromatography on neutral aluminum oxide using methanol:water $(2: 1)$ as mobile phase and analyzed subsequently by HPLC/MS .

High performance liquid chromatography. The analyses were performed using a Agilent 1100 system with SYNERGY ${ }^{\mathrm{TM}}$ MAX-RP columns (Phenomenex, $150 \times 4.6 \mathrm{~mm}$ i.d. and $150 \times 10.0$ $\mathrm{mm}$ i.d., $4 \mu \mathrm{m}$ particle size) at $25^{\circ} \mathrm{C}$. In order to protect the integrity of the analytical and semipreparative column, all analyses were performed with a coupled C-18 guard column. Mobile phase were $10 \mathrm{mM}$ ammonium acetate with $0.08 \%$ formic acid at $\mathrm{pH}=3.5$ and methanol. The elution rates were $10-40 \%$ methanol in $30 \mathrm{~min}$ at a flow rate of $1 \mathrm{ml} / \mathrm{min}$ for the analytical separation and a stepwise change of the composition of organic solvent (A/B (85:15) for 10 min; $\mathrm{A} / \mathrm{B}(95: 5)$ for $5 \mathrm{~min}$; $\mathrm{A} / \mathrm{B}(80: 20)$ for $5 \mathrm{~min})$ at a flow rate of $4.7 \mathrm{ml} / \mathrm{min}$ for the semipreparative separation. Samples were monitored at $254 \mathrm{~nm}$.

Purification and identification. By means of solid phase extraction (Chromafix C18 ec, 900 $\mathrm{mg} / 6 \mathrm{ml}$, Macherey-Nagel) the buffer could be eliminated and compound 4 purely isolated. Structure elucidation was accomplished by spectroscopic methods, including 2D-NMR experiments and comparison with literature data. ${ }^{12}$

LC/MS/MS Analysis. The electrospray ionization (ESI) mass spectra were measured on an Agilent 1100 LC/MSD Trap consisting of an orifice with nitrogen curtain gas and a capillary interface. The conditions of the spray chamber were as follows: ion polarity, positive; capillary voltage, $3500 \mathrm{~V}$; drying gas temperature, $350{ }^{\circ} \mathrm{C}$; nebulizer pressure, $50 \mathrm{psi}$; drying gas flow, $12.001 \mathrm{~min}-1$. LC/MS/MS analyses were performed by using multiple reaction monitoring (MRM). 
Analytical and spectroscopic data for ATAM (1) and 1-hydroxytacrine (2). See Ref. 3. Analytical and spectroscopic data for 9-amino-3,4-dihydroacridin-1(2H)-one (4)

Formula: $\mathrm{C}_{13} \mathrm{H}_{12} \mathrm{~N}_{2} \mathrm{O}$ (212.09). ${ }^{1} \mathrm{H} \mathrm{NMR}\left(400 \mathrm{MHz}, \mathrm{CDCl}_{3}\right) \delta: 10.53\left(1 \mathrm{H}, \mathrm{br}, \mathrm{NH}_{2}\right), 8.94(1 \mathrm{H}$, br, $\left.\mathrm{NH}_{2}\right), 8.08(1 \mathrm{H}, \mathrm{d}, J=8.2 \mathrm{~Hz}, 5-\mathrm{H} / 8-\mathrm{H}), 7.80(1 \mathrm{H}, \mathrm{d}, J=8.1 \mathrm{~Hz}, 5-\mathrm{H} / 8-\mathrm{H}), 7.56(1 \mathrm{H}, \mathrm{t}, J=7.6 \mathrm{~Hz}$, 6-H/7-H), 7.35 (1H, t, J=8.0 Hz, 6-H/7-H), $3.24(2 \mathrm{H}, \mathrm{t}, J=7.4 \mathrm{~Hz}, 2-\mathrm{H}), 2.67$ (2H, t, $J=7.2 \mathrm{~Hz}$, 4-H) 2.16 (2H, m, 3-H). ${ }^{13} \mathrm{C}$ NMR (100 MHz, CDCl3) $\delta: 199.5$ (C-1) q, 161.7 (C-9) q, 156.8 (C4a) q, 139.5 (C-10a) q, 134.1 (C-6), 126.4 (C-7), 123.6 (C-8), 122.3 (C-5), 116.9 (C-9a) q, 104.7 (C-8a) q, 38.8 (C-4), 29.4 (C-2), 20.2 (C-3). ESI-MS m/z: $213[\mathrm{M}+\mathrm{H}]^{+} . \mathrm{MS}^{2} m / z$ : 197 (213 [M $\left.+\mathrm{H}]^{+}-\mathrm{O}^{+}\right) \cdot \mathrm{MS}^{3} \mathrm{~m} / z: 182\left(197[\mathrm{M}+\mathrm{H}]^{+}-\mathrm{NH}^{+}\right) \cdot \mathrm{mp} 237^{\circ} \mathrm{C}\left(\operatorname{Ref} .12 \mathrm{mp} 237-239^{\circ} \mathrm{C}\right)$.

\section{References}

1. Folkers, G.; Kessler, U. Curr. Drug Discovery 2003, 1, 1.

2. Kapková, P.; Heller, E.; Unger, M.; Folkers, G.; Holzgrabe, U. J. Med. Chem. 2005, 48, 7496.

3. Kapková, P.; Heller, E.; Kugelmann, E.; Faber, J.; Bringmann, G.; Kessler, U.; Folkers, G.; Holzgrabe, U. Arch. Pharm. Chem. Life Sci. 2006, 339, 489.

4. Getoff, N.; Ritter, A.; Schwoerer, F.; Bayer, P. Radiat. Phys. Chem. 1993, 41, 797.

5. Shutske, G. M.; Pierrat, F. A.; Cornfeldt, M. L.; Szewczak, M. R.; Huger, F. P.; Bores, G. M.; Haroutunian, V.; Davis, K. L. J. Med. Chem. 1988, 31, 1278.

6. Kapková, P.; Stiefl, N.; Sürig, U.; Engels, B.; Baumann, K.; Holzgrabe, U. Arch. Pharm. Med. Chem. 2003, 336, 523.

7. Harel, M.; Schalk, I.; Ehret-Sabatier, L.; et al. Proc. Natl. Acad. Sci. USA 1993, 90, 9031.

8. Zhao, Q.; Tang, X. C. Eur. J. Pharmacol. 2002, 455, 101.

9. Silman, I.; Harel, M.; Axelsen, P.; Raves, M.; Sussman, J. L. Biochem. Soc. Trans. 1994, 22, 745 .

10. Ploszynska, J.; Kowalski, J.; Sobkowiak, A. Pol. J. Chem. 1998, 72, 2514.

11. Moore, J. A.; Kornreich, L. D. Tetrahedron Lett. 1963, 20, 1277.

12. Drewe, J. A.; Groundwater, P. W. J. Chem. Soc., Perkin Trans. 1 1997, 5, 601. 